\title{
Valor del índice de resistencia arterial medido por Doppler en la función del injerto renal a mediano y largo plazos
}

\author{
Rodolfo E. Torres Serrano ${ }^{1}$, Carlos A. Rebolledoํㅡ, Carlos R. Olivares Algarin 1 , \\ Edgar L. Hernández Botello ${ }^{1}$ y Caterin Delgado Peña ${ }^{1}$ \\ 1 Departamento de Nefrología, Hospital San José, Bogotá, Colombia
}

\section{Resumen}

Objetivo: evaluar la asociación entre el valor del índice de resistencia arterial (IR) medido por doppler al mes postrasplante y la pérdida de injerto. Se reportan los desenlaces secundarios de pérdida mayor o igual al 50\% de la función renal, muerte con injerto funcionante, hallazgos de la biopsia (inflamación, hialinosis, rechazo, fibrosis intersticial - atrofia tubular, IFTA, por sus siglas en inglés).

Métodos: se realizó un estudio de cohorte de 66 pacientes, que recibieron trasplante renal, del Hospital San José de Bogotá, entre octubre de 2007 y abril de 2011; se midió el IR de la arteria hilar por ecografía doppler al mes postrasplante. La mayoría de los pacientes fueron seguidos hasta el segundo año. Se describe la incidencia acumulada de pérdida del injerto renal, pérdida mayor o igual al $50 \%$ de la tasa filtración glomerular; al segundo año postrasplante se reporta el rechazo, IFTA, hialinosis e inflamación documentada en las biopsias de protocolo. Se realizó análisis de asociación en los desenlaces histológicos.

Resultados: solo se reportaron 2 casos de pérdida del injerto renal, pertenecientes al grupo con IR normal (3.8\%). A los 3 años de seguimiento 5 pacientes habían perdido más del $50 \%$ de la TFG respecto a la basal, 4 ocurrieron en los pacientes con IR $<0.8,2$ de ellos (3.7\%) fueron al primer año y solo un paciente con IR $>0.8$ a los 3 años. La mediana de la TFG a los 3 años de seguimiento en ambos grupos es mayor de $60 \mathrm{ml} /$ min. En el primer año de seguimiento 22 (47\%) pacientes con IR normal presentaron IFTA y 7 (54\%) con IR $>0.8$ presentaron IFTA; la hialinosis con un $23 \%$ para IR $>0.8$ y $25.5 \%$ para IR $<0.8$.

Conclusión: los desenlaces de los injertos no solo dependen del IR, hay otros factores que pueden influir como es el tiempo de isquemia fría, esquema de tratamiento inmunosupresor de inducción y mantenimiento, grado de incompatibilidades entre donante, receptor.

Cambios histopatológicos como IFTA, inflamación, hialinosis, se observaron en cualquiera de los 2 grupos, lo que sugiere que hay otros factores más fuertes que el IR, que se correlaciona con la aparición de estos hallazgos.

Palabras clave: Injerto renal, Índice de resistencia arterial, Tasa de Filtración Glomerular.

\section{Correlation between arterial resistance índex by doppler in renal graft function}

\begin{abstract}
Objective: To assess the association between the value of arterial resistance index (RI) measured by Doppler month post transplant and graft loss. Secondary outcomes of greater than or equal to $50 \%$ loss of renal
\end{abstract}


function, death with a functioning graft biopsy findings (inflammation, hyalinosis, rejection, IFTA) are reported.

Methods: A cohort study of 66 patients who received a kidney transplant at the Hospital San José de Bogotá, between October 2007 and April 2011 was performed; arterial resistance index hilar artery doppler ultrasound month post-transplant was measured. Most patients were followed until the second year. The cumulative incidence of graft loss is described, lost greater than or equal to $50 \%$ of glomerular filtration rate; tubular atrophy (IFTA), hyalinosis and documented inflammation in protocol biopsies - the second year transplant rejection, interstitial fibrosis is reported. Association analysis was performed in histological outcomes.

Results: Two cases of graft loss was reported and all belonging to the group with normal (3.8\%) IR. At 3 -year follow-up five patients had lost more than $50 \%$ of GFR from baseline, where four occurred in patients with $\mathrm{RI}<0.8$, two (3.7\%) were the first year and only one patient with index blood $>0.8$ resistance to 3 years. Median GFR at 3 years follow-up in both groups was greater than $60 \mathrm{ml} / \mathrm{min}$. In the first year of follow-up $22(47 \%)$ patients with normal IR presented interstitial fibrosis and tubular atrophy (IFTA), and seven (54\%) with IR $>0.8$ presented IFTA; hyalinosis with $23 \%$ for IR $>0.8$, and $25.5 \%$ for IR $<0.8$.

Conclusion: The outcome of the grafts not only depend on the index of arterial resistance, other factors that may influence such as cold ischemia time, immunosuppressive treatment scheme for induction and maintenance, grade mismatches between donor, recipient.

Histopathological changes as IFTA, inflammation, hyalinosis was observed in either group, which suggests that other factors strongest IR, which correlates with the appearance of these findings.

Keywords: renal graft, arterial resistance index, glomerular filtration rate

\section{Introducción}

E 1 trasplante renal se constituye, hoy día, en la mejor opción terapéutica para los pacientes con insuficiencia renal crónica, en términos de supervivencia y calidad de vida ${ }^{1 ;}$ grandes avances en los esquemas inmunosupresores de inducción y mantenimiento con micofenolato sódico y mofetil, calcineurinicos, inhibidores M-tor, han incrementado la sobrevida del paciente y del injerto renal durante los años postrasplante, desde un $70 \%$ reportado a principios de los años 90 hasta $90 \%$ hoy en día ${ }^{1,2}$. A pesar de esto la sobrevida renal a largo plazo, es decir, luego del primer año del trasplante, no ha mejorado de manera paralela y cerca del 50 al $80 \%$ de los injertos renales se pierden dentro de los 10 primeros años, pero lo que no tenemos claro es si hay algún método de predecir estos desenlaces ${ }^{3,4}$.

La causa más frecuente de etiología no renal de la pérdida del injerto, sigue siendo la enfermedad cardiovascular ${ }^{5}$, seguida del desarrollo de neoplasias malignas precipitadas por algunos de los esquemas inmunosupresores ${ }^{6}$. Otras causas son las alteraciones renales irreversibles secundarias a procesos inmunológicos, que se pueden observar en la biopsia renal, tales como: IFTA y su correlación con el grado de alteración (IFTA GI alteración del 5 al 25\%, IFTA GII alteración del 26 al 50\%, IFTA GIII mayor del $50 \%)^{7}$.

Para la identificación de los receptores de injertos renales que están en riesgo de pérdida tardía, se han propuesto estrategias diagnósticas que conduzcan a intervenciones terapéuticas, diseñándose en la literatura múltiples factores de riesgo con valores predictivos limitados ${ }^{8,9}$. Uno de estos factores es la utilización del IR, del injerto renal, medido por ecografía doppler como factor predictor de disfunción tardía del mismo ${ }^{8}$. En la literatura se documentan publicaciones que evalúan el valor del IR mayor o igual a 0.8 como un factor de riesgo de disfunción del injerto con resultados alentadores en cuanto a su papel en la capacidad de establecer el pronóstico de la función del mismo a largo plazo; sin embargo, los estudios presentan algunas limitantes metodológicas ${ }^{10,11}$.

Existen publicaciones en las cuales se discute que quizás el IR sea un predictor de enfermedad ateroesclerótica o más bien refleja una compleja integración entre resistencia periférica, distensibilidad y pulsatibilidad arterial, que se asocian con otros factores de riesgo tradicionales cardiovasculares; mas no un marcador específico de daño renal, por lo tanto su 
utilidad clínica en el diagnóstico de rechazo o pérdida del injerto sería limitada ${ }^{12}$.

Se ha explorado que la mejor comprensión de la relación IR/nefropatía crónica del injerto, está mediante la correlación de las mediciones ecográficas y el examen histológico de las biopsias renales ${ }^{13,14}$.

\section{Objetivos}

El objetivo de este estudio fue evaluar la asociación entre el valor del IR medido por doppler al primer mes postrasplante y la pérdida de injerto. Se reportan los desenlaces secundarios, de pérdida del $50 \%$ o más de la función renal, muerte con injerto funcionante y hallazgos de la biopsia renal: inflamación, rechazo, hialinosis y el IFTA.

\section{Métodos}

\section{Pacientes y diseño del estudio}

El protocolo fue aprobado por el Comité de Inves-tigaciones y Ética de la Fundación Universitaria de Ciencias de la Salud - Hospital San José.

Se trata de una cohorte prospectiva con seguimiento a 3 años, se reclutaron todos los casos consecutivos que cumplieron los criterios de inclusión: mayores de 18 años a quienes se les realizó un trasplante renal en el Hospital San José de Bogotá y tuvieran medición del IR al primer mes postrasplante. Se excluyeron pacientes que persistían en función lenta (diuresis menor a $100 \mathrm{cc} /$ hora o descenso menor al $30 \%$ de la creatinina basal sin requerimiento de diálisis) o función retardada (requerimiento de diálisis postrasplante, secundario a disfunción del injerto renal) por más de cuatro semanas postrasplante y la presencia de complicaciones mecánicas que no se hubieran corregido al momento de la medición del IR.

Se realizó ultrasonografía doppler color en la arteria hiliar al cumplir el primer mes postrasplante, eligiendo este tiempo para asegurar la recuperación de la función del injerto de cualquier tipo de injuria aguda. Todos los procedimientos fueron realizados con el equipo Toshiba Nemio 20, utilizando un transductor de 2 - $4 \mathrm{MHz}$, en el servicio de radiología.
El IR se obtuvo de la siguiente fórmula: velocidad pico sistólica - velocidad pico al final de la diástole / velocidad pico sistólica. Se estratificó como menor de 0.8 y mayor o igual a 0.8 , interpretado como normal y anormal, respectivamente ${ }^{5,6}$.

La tasa de filtración glomerular se calcula al primer mes (basal), al primer año por medio de la utilización de la fórmula MDRD, con seguimiento anual de la tasa de filtración glomerular por 3 años. Por protocolo del servicio, a partir del año 2007 todos los pacientes son llevados a biopsia del injerto entre el sexto mes y el primer año postrasplante, a menos que tengan alguna contraindicación.

\section{Análisis estadístico}

El análisis se realizó con el programa $S$ tata ${ }^{\circledR}$ versión 10. Mediante la estadística descriptiva se resumen las variables cuantitativas con medidas de tendencia central y dispersión. Se presentan tablas y gráficos de cajas y bigotes para describir la evolución de la tasa de filtración glomerular. Las variables cualitativas se presentan como frecuencias absolutas y porcentajes. Se reporta la incidencia acumulada de pérdida del injerto renal, pérdida del $50 \%$ de la TFG y muerte; al segundo año postrasplante se reporta el rechazo, IFTA, hialinosis e inflamación documentada en las biopsias de protocolo. Se realizó análisis de asociación en los desenlaces histológicos.

\section{Resultados}

Se analizan 66 casos ingresados a la cohorte entre octubre de 2007 y abril de 2011. Las características demográficas basales de la población según el IR se describen en la tabla 1.

La razón mujer / hombre es 2:1. La edad promedio en los pacientes con IR menor de 0.8 fue de 42.3 años e IR $>0.8$ fue de 49.2 años. La proporción de pacientes con presencia de hipertensión arterial y diabetes mellitus como etiología de insuficiencia renal es menor entre quienes tuvieron IR normal, $26.4 \%$ y $9.4 \%$, respectivamente, en contraste con $30.7 \%$ y $15.3 \%$ en la población con IR anormal. La proporción de pacientes con causa no conocida de insuficiencia renal crónica fue mayor entre quienes tuvieron IR normal (37.7\% vs. 30.7\%). 


\section{Tabla 1}

Características demográficas y clínicas según el índice de resistencia arterial (IR)

\begin{tabular}{|c|c|c|c|c|}
\hline & \multicolumn{2}{|c|}{ IR $<0.8$} & \multicolumn{2}{|c|}{ IR $\geq \mathbf{0 . 8}$} \\
\hline & $\mathbf{n}$ & $(\%)$ & $\mathbf{n}$ & $(\%)$ \\
\hline & 53 & $(80.3)$ & 13 & $(19.7)$ \\
\hline Edad (promedio DE*) & 42.3 & $(13.0)$ & 49.2 & $(13.0)$ \\
\hline Mínimo - máximo & & $(18-65)$ & & $(26-74)$ \\
\hline \multicolumn{5}{|l|}{ Sexo, n $(\%)$} \\
\hline Masculino & 17 & $(32.1)$ & 4 & $(30.1)$ \\
\hline \multicolumn{5}{|c|}{ Etiología enfermedad renal crónica en nativos, n (\%) } \\
\hline Hipertensión arterial & 14 & $(26.4)$ & 4 & $(30.7)$ \\
\hline Diabetes mellitus & 5 & $(9.4)$ & 2 & $(15.3)$ \\
\hline Enfermedad glomerular & 7 & $(13.2)$ & 0 & 0 \\
\hline Enfermedad poliquística & 0 & 0 & 1 & $(7.6)$ \\
\hline Lupus eritematoso sistémico & 2 & $(3.7)$ & 1 & (7.6) \\
\hline Nefritis intersticial & 0 & 0 & 0 & 0 \\
\hline Otras & 5 & $(9.4)$ & 1 & $(7.6)$ \\
\hline Desconocida & 20 & $(37.7)$ & 4 & $(30.7)$ \\
\hline Isquemia fría, horas mediana (RIQ) & 12 & $(8,14)$ & 12 & $(12,13)$ \\
\hline Menor de 12 horas n (\%) & 28 & $(52.8)$ & 7 & $(53.8)$ \\
\hline 12- 24 horas n (\%) & 25 & $(47.2)$ & 6 & $(46.2)$ \\
\hline \multicolumn{5}{|l|}{ Tipo de donante $\mathrm{n}(\%)$} \\
\hline Cadavérico & 47 & $(88.7)$ & 13 & $(100)$ \\
\hline Vivo & 6 & $(11.3)$ & 0 & \\
\hline Donante marginal n $(\%) \ddagger$ & 3 & $(5.6)$ & 3 & $(23.1)$ \\
\hline \multicolumn{5}{|l|}{ Miss match de HLA $\S, \mathrm{n}(\%)$} \\
\hline 0 & 1 & $(1.8)$ & 0 & \\
\hline 1 & 2 & $(3.7)$ & 0 & \\
\hline 2 & 8 & $(15.9)$ & 1 & $(7.6)$ \\
\hline 3 & 16 & $(30.1)$ & 5 & $(38.4)$ \\
\hline 4 & 16 & $(30.1)$ & 4 & $(30.7)$ \\
\hline 5 & 7 & $(13.2)$ & 2 & $(15.3)$ \\
\hline 6 & 3 & $(5.6)$ & 1 & $(7.6)$ \\
\hline \multicolumn{5}{|l|}{ Inducción $\|, \mathrm{n}(\%)$} \\
\hline Timoglobulina & 18 & (34) & 5 & $(38.5)$ \\
\hline Basiliximab & 35 & $(66)$ & 8 & $(61.5)$ \\
\hline \multicolumn{5}{|l|}{ Tratamiento inmunosupresor $\mathbf{q}, \mathrm{n}(\%)$} \\
\hline Ciclosporina, micofenolato, esteroides & 12 & $(22.7)$ & 5 & $(38.5)$ \\
\hline
\end{tabular}


Continuación Tabla 1.

\begin{tabular}{|l|c|c|c|c|}
\hline & \multicolumn{2}{|c|}{ IR $<\mathbf{0 . 8}$} & \multicolumn{2}{|c|}{ IR $\geq \mathbf{0 . 8}$} \\
\hline & $\mathbf{n}$ & $\mathbf{( \% )}$ & $\mathbf{n}$ & $\mathbf{( \% )}$ \\
\hline Tacrolimus, micofenolato, esteroides & $\mathbf{5 3}$ & $(80.3)$ & $\mathbf{1 3}$ & $(19.7)$ \\
\hline Función inmediata **, n (\%) & 41 & $(77.4)$ & 8 & $(61.5)$ \\
\hline Función lenta ††, n (\%) & 39 & $(73.6)$ & 9 & $(69.2)$ \\
\hline Función retardada †ł, n (\%) & 6 & $(11.3)$ & 1 & $(7.6)$ \\
\hline Complicaciones al mes ***, n (\%) & 8 & $(15)$ & 3 & $(23.1)$ \\
\hline Mecánicas & 15 & $(28.3)$ & 3 & $(23.1)$ \\
\hline Vasculares & 3 & $(5.7)$ & 1 & $(7.7)$ \\
\hline Necrosis tubular aguda & 1 & $(1.9)$ & 0 & $(0)$ \\
\hline Rechazo primer mes & 13 & $(24.5)$ & 3 & $(23.1)$ \\
\hline
\end{tabular}

El tiempo mediano de isquemia fría para toda la cohorte fue de 12 horas (RIQ 8-14). La mayoría de los injertos renales fueron provenientes de donantes cadavéricos, en $89 \%$ para el grupo de IR $<0.8$ y $100 \%$ para IR $>0.8$, con presencia de 3 donantes marginales en cada grupo.

El grado de incompatibilidades del HLA entre donante y receptor que más predominó en los dos grupos, fue 3 y 4 en el $60.2 \%$ para IR normal y $69.1 \%$ para IR anormal.

El esquema de inducción más utilizado en los dos grupos de IR fue el basiliximab en $64 \%$. El tratamiento inmunosupresor basado en tacrolimus, micofenolato y corticoide se usó en 41 casos $(77.3 \%)$ de los pacientes con IR normal y en 8 casos $(61.5 \%)$ de los pacientes con IR anormal.

La mayoría de los pacientes en los dos grupos tuvieron función inmediata del injerto, 39 sujetos (73.6\%) con IR normal y 9 sujetos (69.2\%) con IR anormal. Función lenta del injerto se presentó en 6 pacientes con IR normal $(11.3 \%)$ y un paciente con IR anormal $(7.6 \%)$. La función retardada del injerto estuvo presente en $15 \%$ los pacientes con IR normal y en $23.1 \%$ de los pacientes con IR anormal. La necrosis tubular aguda fue la causa más frecuente de función retardada en los dos grupos.

En los pacientes con IR normal se observaron 3 complicaciones mecánicas $(5.7 \%)$ y en los pacientes con
IR anormal se observó una complicación mecánica (7.7\%), todas secundarias a fístulas ureterovesicales; cabe resaltar que en el momento de la medición del IR al mes postrasplante estas fístulas ya estaban corregidas. Se presentó un caso (1.9\%) de complicación vascular correspondiente a una alteración a nivel de la anastomosis vascular venosa con función retardada del injerto y requiriendo warfarina de forma indefinida como tratamiento, motivo por el cual no se le realizó biopsia renal.

Se identificaron 2 eventos de rechazo en el primer mes postrasplante pertenecientes a los pacientes con IR normal (3.8\%) con respuesta adecuada a esteroides y sin pérdida de la función renal.

Los desenlaces según el IR están representados en la tabla 2. Se presentó muerte con injerto funcionante en 3 casos con IR normal (5.6\%), 1 secundario a proceso séptico de origen abdominal y 2 por causa cardiovascular, todos ocurrieron antes de cumplir el año postrasplante, mientras que en los pacientes con IR anormal solo se evidenció un caso (7.7\%) secundario a un evento cardiovascular y también antes del primer año postrasplante. Se reportan 2 casos de pérdida del injerto renal $(3.8 \%)$ pertenecientes al grupo con IR normal; uno corresponde al desarrollo de una nefropatía por polioma virus. No se encontró ninguna pérdida del injerto en el grupo con IR anormal. La pérdida de más del $50 \%$ de la TFG respecto a la 


\section{Tabla 2}

\begin{tabular}{|c|c|c|c|c|}
\hline \multicolumn{5}{|c|}{ Desenlaces según índice de resistencia arterial } \\
\hline & \multicolumn{2}{|c|}{ IR $<0.8$} & \multicolumn{2}{|c|}{ IR $\geq 0.8$} \\
\hline & $\mathbf{n}$ & 53 & $\mathbf{n}$ & 13 \\
\hline Muerte con injerto funcionante $\mathbf{n}(\%)$ & 3 & $(5.7)$ & 1 & (7.7) \\
\hline Pérdida del injerto * n (\%) & 2 & $(3.8)$ & 0 & \\
\hline Pérdida TFG $\geq 50 \%$ a 1 año n (\%) & 2 & (3.7) & 0 & \\
\hline \multirow[t]{2}{*}{ Pérdida TFG $\geq \mathbf{5 0 \%}$ a 2años n (\%) } & 2 & $(3.7)$ & 0 & \\
\hline & 0 & & 1 & (7.7) \\
\hline \multicolumn{5}{|l|}{ TFG mes postrasplante MDRD $†$} \\
\hline MDRD mediana (RIQ) & 64 & $(48.3-72.3)$ & 58 & $(49.8-70.6)$ \\
\hline Mínimo-máximo & 12.7 & $(138.5)$ & 22.7 & $(100.1)$ \\
\hline \multicolumn{5}{|l|}{ TFG 1 año postrasplante MDRD $\dagger$} \\
\hline MDRD mediana (RIQ) & 67.8 & $(56.9-86.2)$ & 64.4 & $(59.1-82.8)$ \\
\hline Mínimo-máximo & 18.7 & $(138.5)$ & 32.1 & $(102)$ \\
\hline \multicolumn{5}{|l|}{ TFG a 3 años postrasplante MDRD $†$} \\
\hline MDRD mediana (RIQ) & 65.2 & $(48.9-70.7)$ & 64.6 & $(60.1-71.5)$ \\
\hline Mínimo- máximo & 44 & (118) & 25 & $(105)$ \\
\hline \multicolumn{5}{|l|}{ Severidad IFTA al primer año de trasplante $\ddagger$} \\
\hline I & 15 & $(32.6)$ & 6 & $(46.1)$ \\
\hline II & 5 & $(10.8)$ & 1 & (7.7) \\
\hline III & 2 & $(4.35)$ & 0 & 0 \\
\hline \multicolumn{5}{|l|}{ Severidad IFTA hasta 2 años de trasplante $\S$} \\
\hline I & 22 & $(42.3)$ & 8 & $(61.5)$ \\
\hline II & 11 & $(21.1)$ & 2 & $(15.3)$ \\
\hline III & 1 & $(1.9)$ & 0 & \\
\hline Cualquier rechazo del injerto renal al primer año & 13 & $(24.5)$ & 3 & (23.1) \\
\hline Cualquier rechazo del injerto renal al segundo año§ & 16 & (31.4) & 5 & $(38.4)$ \\
\hline Hialinosis§ & 13 & $(25.5)$ & 3 & (23) \\
\hline Inflamación § & 31 & $(61)$ & 8 & ( 61.5) \\
\hline Conversión a inhibidores del complejo M-tor n (\%) & 14 & $(26.4)$ & 6 & $(46.1 \%)$ \\
\hline
\end{tabular}

* Seguimiento a dos años de 53 pacientes. † Mediana tasa de filtración glomerular calculada por MDRD 1 año 64 pacientes de seguimiento, al 3 año con 34 pacientes de seguimiento. ¥ Clasificación BANFF 2009 Leve $<25 \%$ del área cortical. Moderada 26-50\% Severa $>50 \%$. Datos disponibles para 43 pacientes con IR normal y 12 pacientes con IR anormal. §Para biopsias de 2do año la hialinosis, inflamación, rechazo son datos disponibles para 51 pacientes con IR normal y 13 para IR anormal.

basal al año de seguimiento se presentó en 2 pacientes $(3.8 \%)$ con IR normal; con un total de 5 pacientes a los 3 años de seguimiento, de los cuales solo uno se presentó con IR $>0.8$ y ocurrió a los 3 años.
En el primer año de seguimiento $22(47 \%)$ pacientes con IR normal presentaron IFTA, la mayor parte de ellos fueron clasificados como IFTA de severidad grado I en el 32.6\%. Entre los pacientes con 
IR anormal, 7 (54\%) presentaron IFTA clasificada como grado I en el $46.1 \%$. Después del primer año de seguimiento se realizaron biopsias de protocolo evidenciándose un aumento en el IFTA global: 33 pacientes $(65 \%)$ en los pacientes con IR $<0.8$ y 10 pacientes $(77 \%)$ con IR $>0.8$; además, se observó un incremento mayor del $50 \%$ en el grado de IFTA clasificada como grado II con respecto a la primera biopsia de protocolo para ambos grupos, sin cambios en IFTA grado III.

Se identificó cualquier tipo rechazo en las biopsias de protocolo (la mayoría de características subclínicas) al primer mes, primer año y después del año. Hasta el primer año 13 pacientes $(24.5 \%)$ con IR normal y 3 pacientes $(23 \%)$ con IR anormal habían presentado algún tipo de rechazo del injerto. Pero con el seguimiento de las biopsias de protocolo al segundo año, se observa que la incidencia de rechazo en ambos grupos aumentó a $31.4 \%$ con IR $<0.8$ y $38.4 \%$ a los de IR $>0.8$.

En esta cohorte se observó un porcentaje casi similar de hialinosis en los dos grupos de pacientes, con un $23 \%$ para IR $>0.8$ y $25.5 \%$ para IR $<0.8$; además 2 de los 3 pacientes convertidos a $\mathrm{M}$ - tor en el grupo $\geq 0.8$ presentaron hialinosis y 5 de los 13 en el grupo de IR $<0.8$ la presentó.
La presencia de algún grado de inflamación intersticial se evidenció en el $61 \%$ de los pacientes con IR $<0.8$ y en $61.5 \%$ de los pacientes IR mayor de 0.8 , con mayor severidad de la inflamación en el grupo de IR $<0.8$.

Se realizaron 20 conversiones a inhibidores del complejo M-tor, 14 con IR normal (26.4\%) y 6 con IR anormal (46.1\%). Doce de todas las conversiones se efectuaron durante los primeros 6 meses postrasplante. De los pacientes con IR normal convertidos a inhibidores de M-tor, 2 (3.8\%) perdieron más del $50 \%$ de la TFG al primer año, el primero de ellos al tercer mes por una nefropatía por polioma virus que conllevó a la pérdida de la función del injerto a los 10 meses postrasplante; el segundo paciente por un rechazo humoral. De los pacientes con IR anormal ninguno perdió más del $50 \%$ de la TFG al 1 año y solo 1 paciente convertido a inhibidor del M-tor presentó disminución del 50\% de la TFG ocurriendo al tercer año de seguimiento. La hialinosis se presentó en 5 pacientes con IR $<0.8$ y 2 pacientes con IR $>0.8$. Doce pacientes presentaron IFTA, el grado de severidad III, la obtuvieron dos pacientes todos con IR normal. No se presentaron rechazos de los injertos renales (tabla 3 ).

Tabla 3

Desenlaces de los pacientes convertidos a inhibidores del complejo M-tor

\begin{tabular}{|l|c|c|}
\hline & IR $<\mathbf{0 . 8}$ & IR $\geq \mathbf{0 . 8}$ \\
\cline { 2 - 3 } & $\mathbf{n}(\%)$ & $\mathbf{n}(\%)$ \\
\cline { 2 - 3 } & $\mathbf{5 3}$ & $\mathbf{1 3}$ \\
\hline Número de pacientes convertidos n(\%) & $14(26.4)$ & $6(46.1)$ \\
\hline Pérdida TFG $\geq \mathbf{5 0 \%}$ al año* $\mathbf{n}(\%)$ & $3(5.6 \%)$ & 0 \\
\hline Muerte con injerto funcionante $\mathbf{n}(\%)$ & 0 & $1(7.6 \%)$ \\
\hline Pérdida del injerto n (\%) & $1(1.8 \%)$ & \\
\hline Severidad IFTA al primer año de trasplante + & & $3(23 \%)$ \\
\hline I & $5(9.4 \%)$ & 0 \\
\hline II & $2(3.7 \%)$ & $1(7.6 \%)$ \\
\hline III & $2(3.7 \%)$ & $2(3.8)$ \\
\hline Hialinosis & $5(9.4)$ & 0 \\
\hline Rechazo del injerto renal al primer año & 0 & \\
\hline
\end{tabular}

*Incidencias acumuladas. † Datos disponibles para 20 pacientes 
Las figuras 1 y 2 muestran los cambios en la tasa de filtración glomerular durante el primero y el segundo año, respectivamente. Se puede observar como la mediana de la tasa de filtración glomerular basal es menor en el grupo de IR $>0.8$ casi $6 \mathrm{ml} / \mathrm{min}$ y, posteriormente, en los 2 grupos permanece estable durante los dos años de seguimiento. No parece existir un momento en el tiempo donde se produzcan cambios representativos de la tasa de filtración glomerular.

La mediana de la tasa de filtración glomerular calculada por MDRD en los 2 años de seguimiento con 54 pacientes y a los 3 años con 34 pacientes sigue siendo mayor de $60 \mathrm{ml} / \mathrm{min}$ (tabla 4).

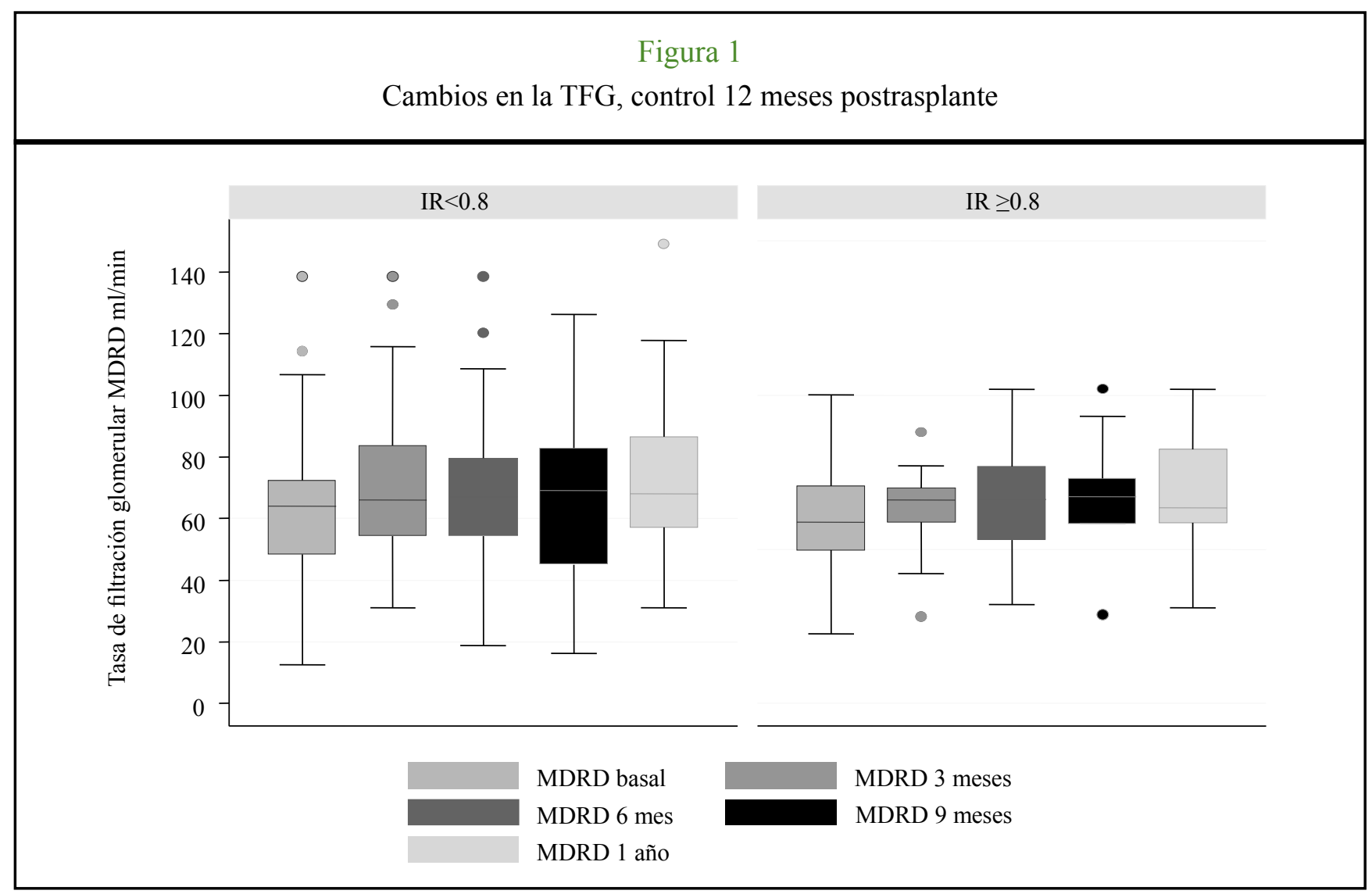

\section{Tabla 4}

Cambios en la tasa de filtración glomerular (MDRD) según índice de resistencia

\begin{tabular}{|c|c|c|c|c|c|c|c|c|c|}
\hline & & $\begin{array}{l}\text { TFG basal } \\
*\end{array}$ & $\begin{array}{l}\text { TFG } 3 \\
\text { meses } †\end{array}$ & $\begin{array}{l}\text { TFG } 6 \\
\text { meses } \ddagger\end{array}$ & $\begin{array}{l}\text { TFG } 9 \\
\text { meses } §\end{array}$ & $\begin{array}{l}\text { TFG } 12 \\
\text { meses } \|\end{array}$ & $\begin{array}{l}\text { TFG } 24 \\
\text { meses } 9\end{array}$ & $\begin{array}{l}\text { TFG } 36 \\
\text { meses ** }\end{array}$ & $\begin{array}{l}\text { TFG } 48 \\
\text { meses†† }\end{array}$ \\
\hline IR $<0.8$ & $\begin{array}{l}\text { Mediana } \\
\text { (RIQ) }\end{array}$ & $\begin{array}{l}64 \\
(48.3-72.3)\end{array}$ & $\begin{array}{l}66 \\
(51.3-83.6)\end{array}$ & $\begin{array}{l}67.1 \\
(54.4-79.6)\end{array}$ & $\begin{array}{l}69.2 \\
(45.1-82.8)\end{array}$ & $\begin{array}{l}67.8 \\
(56.9-86.2)\end{array}$ & $\begin{array}{l}71.3 \\
(61.3-83.8)\end{array}$ & $\begin{array}{l}65.2 \\
(48.9-70.7)\end{array}$ & $\begin{array}{l}67.8 \\
(56.5-78.1)\end{array}$ \\
\hline $\mathrm{IR} \geq 0.8$ & $\begin{array}{l}\text { Mediana } \\
\text { (RIQ) }\end{array}$ & $\begin{array}{l}58.8 \\
(49.8-70.6)\end{array}$ & $\begin{array}{l}66.1 \\
(58.8-70)\end{array}$ & $\begin{array}{l}66.3 \\
(53.1-77)\end{array}$ & $\begin{array}{l}66.9 \\
(58.4-73)\end{array}$ & $\begin{array}{l}64.4 \\
(59.1-82.8)\end{array}$ & $\begin{array}{l}68.5 \\
(66.6-70.4)\end{array}$ & $\begin{array}{l}64.6 \\
(60.1-71.5)\end{array}$ & $\begin{array}{l}67 \\
(63.2-70.5)\end{array}$ \\
\hline
\end{tabular}

* Datos disponibles para 53 pacientes. †Datos disponibles para 51 pacientes con IR normal y 13 pacientes con IR anormal. $\ddagger$ Datos disponibles para 45 pacientes con IR normal y 13 pacientes con IR anormal. §Datos disponibles para 39 pacientes con IR normal y 10 pacientes con IR anormal. || Datos disponibles para 45 pacientes con IR normal y 13 pacientes con IR anormal. ๆ Datos disponibles para 43 pacientes con IR normal y 11 pacientes con IR anormal. **Datos disponibles para 26 pacientes con IR normal, y 8 con IR anormal. †† Datos disponibles para 9 pacientes con IR normal y 4 pacientes con IR anormal. 


\section{Figura 2}

Cambios en la TFG, control 24 meses postrasplante
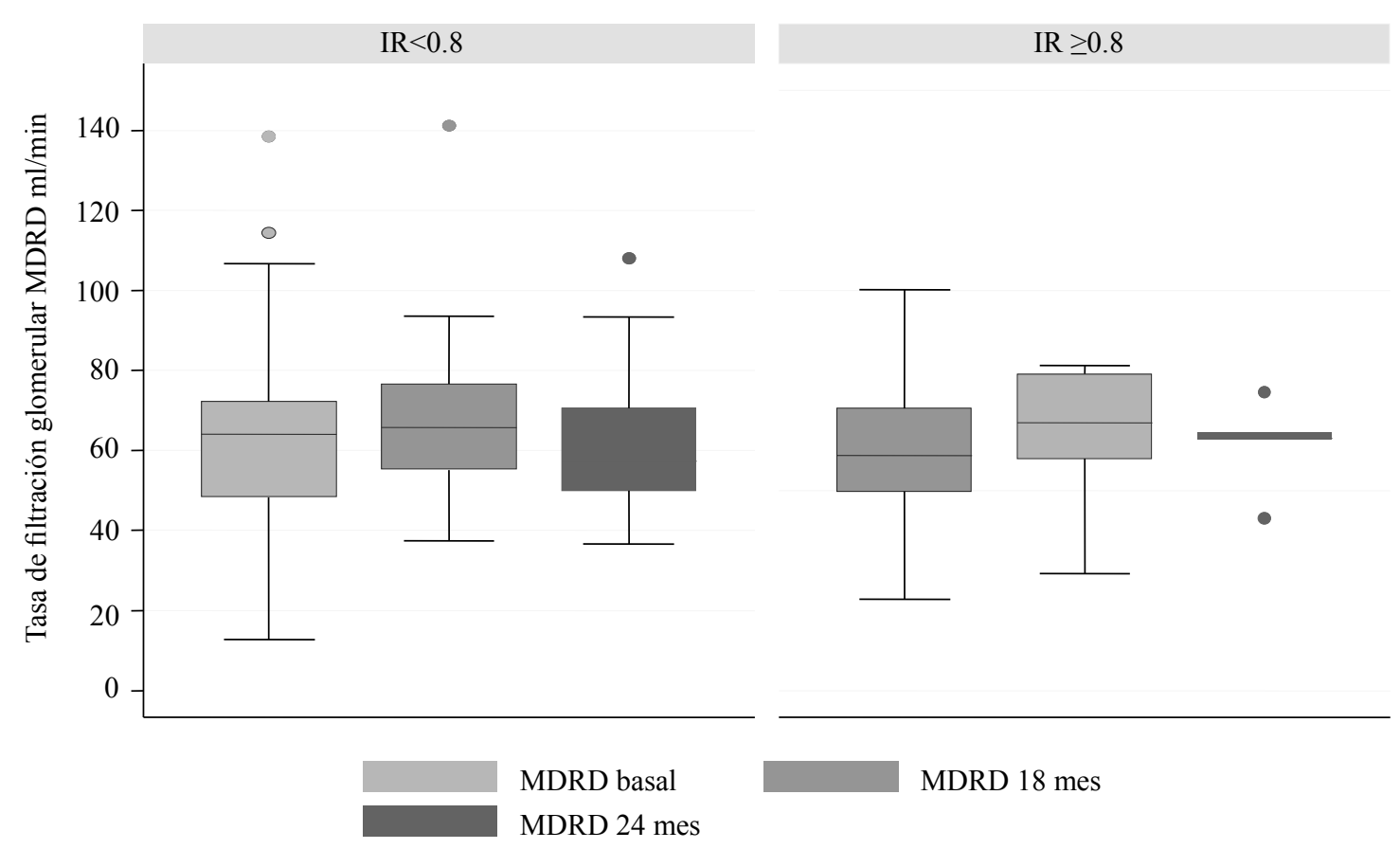

El $32.4 \%$ de los pacientes con IR normal y el $25 \%$ con IR anormal no perdieron más del $30 \%$ de la TFG respecto a la basal al año de seguimiento. Estos mismos desenlaces en los 2 grupos se muestran en la figura 3.

En el seguimiento se presentaron 3 casos de nefropatía por polioma virus, uno de ellos antes de cumplir un año de trasplante y perdió la función del injerto, los otros 2 mantienen niveles séricos elevados de creatinina pero no han requerido terapia de reemplazo renal.

\section{Discusión}

Grandes avances en los esquemas de inmunosupresión han permitido un mayor incremento en la sobrevida del paciente y del injerto renal, en el primer año postransplante, desde un $70 \%$ reportado a principios de los años 90 hasta un $90 \%$ hoy en día ${ }^{1,2}$, sin embargo, la sobrevida a largo plazo no ha mejorado de manera paralela y entre el 50 y el $80 \%$ de los injertos renales se pierden dentro de los 10 primeros años. Esto ha conllevado a que se implementen estrategias diagnósticas tempranas con el fin de poder diagnosticar a los pacientes con alto riesgo de perder el injerto renal. La controversia se suscita en poder encontrar la herramienta más oportuna, temprana y efectiva que nos ayude a tomar decisiones terapéuticas que sean de beneficio a largo plazo.

En la literatura hay varios estudios que evalúan el valor del IR como factor de riesgo de función tardía del injerto, con resultados alentadores en cuanto a su papel en la capacidad de establecer el pronóstico de la función del injerto a largo plazo. Son varias las publicaciones que están a favor y en contra, que evalúan la relación entre IR y la función del injerto renal en el tiempo, esto con el objetivo de tener una medida no invasiva que sirva como predictor de sobrevida con impacto clínico $8,9,15,16$.

Radermacher realizó un seguimiento a 36 meses con 601 pacientes trasplantados, evidenciando que quienes tenían un IR $>0.8$ tenían un riesgo relativo (RR) 


\section{Figura 3}

Porcentaje de pacientes con aumento y pérdida de la TFG, control 12 meses postrasplante

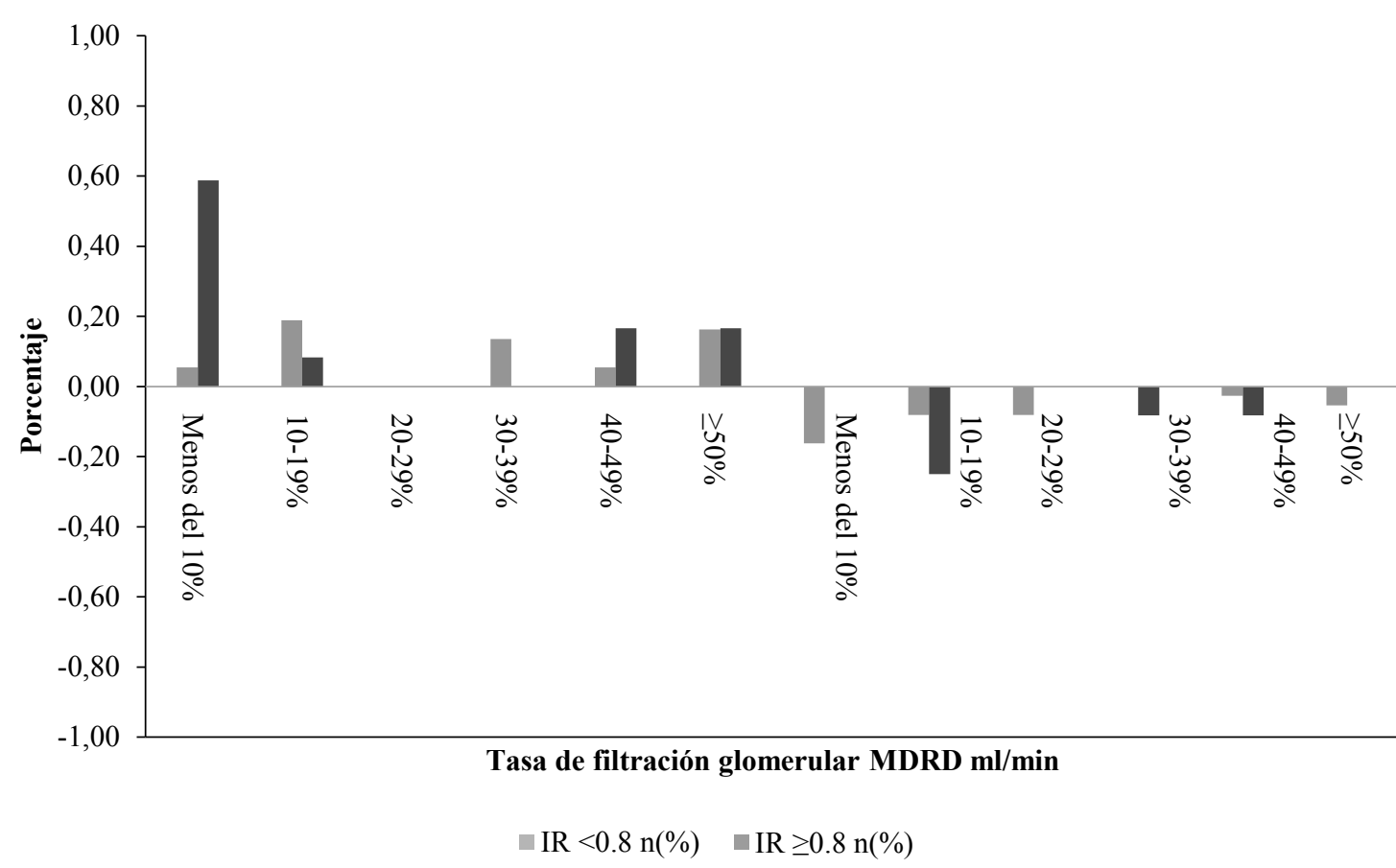

de 9.1 (IC 6.6-12.1) para pérdida del injerto en 2.5 años $^{11}$, y señala como conclusión que los IR mayores de 0.8 se correlacionan con un alto riesgo de pérdida de los injertos renales, aun en el análisis multivariado; sin embargo, la medición del IR no se realizó en un punto estipulado, solo en el seguimiento, lo cual puede conllevar a conclusiones erróneas de los resultados.

Saracino en un estudio retrospectivo con 76 pacientes y seguimiento a 100 meses disminuye el punto de corte del IR, con resultados que demuestran que aquellos con valores mayores a 0.63 presentaron un deterioro de la TFG del $24 \%$ con RR de 3.77 (IC 1.5-12) y una estrecha correlación con la edad del donante y el receptor ${ }^{15}$.

Nezami, años más tarde, también describe la misma asociación significativa entre IR y niveles de creatinina sérica medidos al primer mes postrasplante en una cohorte retrospectiva de 273 pacientes; concluyendo que la presencia de estenosis en la arteria renal puede dar de manera falsa valores normalizados ${ }^{13}$. A pesar de estos estudios, otros autores discuten el hecho de que el IR pueda tener valor clínico en relación con la función del injerto en el tiempo; Kebkowska no evidenció relación con la TFG al mes de trasplante en una cohorte prospectiva de 41 pacientes, IR entre 0.74-0.76 ${ }^{14}$. Bergman en un estudio prospectivo de 36 meses, evaluó a 200 pacientes trasplantados encontrando correlación de la tasa filtración glomerular con la edad, la presencia de diabetes o enfermedad cardiovascular y, particularmente, con los niveles de ADMA (dimetil arginina asimétrica), una toxina urémica bien descrita como causa de hipertensión arterial en pacientes urémicos; sin embargo, no hubo asociación con el IR ${ }^{17}$.

Tratando de establecer un vínculo entre IR anormal y la TFG que explique la relación encontrada, muy pocos estudios han abordado los hallazgos de las biopsias renales de protocolo y su asociación con el IR. Vallejo, en un estudio retrospectivo que incluía 87 pacientes describe que los injertos con IR más altos (0.85) mostraban en la patología un mayor puntaje de glomerulitis; sin embargo no evidenció correlación entre IR y nefropatía crónica del injerto ${ }^{18}$. 
Kirkpantur ${ }^{19}$, en una serie de pacientes trasplantados encontró que un IR mayor a 0.75 se asoció a mayor esclerosis glomerular, fibrosis intersticial y arteriosclerosis severa. La evidencia en este tópico es muy limitada, pero se espera que con la realización de biopsias de protocolo, como parte del seguimiento en pacientes trasplantados, se identifiquen más avances.

El incremento del IR al mes se sigue observando en nuestros pacientes con edades mayores, diabéticos e hipertensos, este fenómeno tal vez asociado a la mayor presencia de arteriosclerosis.

La minoría de la población trasplantada en nuestro centro es proveniente de donante vivo y al igual que en la literatura ${ }^{20}$ no se encontró en este grupo de pacientes aumento del IR, esto probablemente relacionado con factores quirúrgicos, menores tiempos de isquemia fría y menos incompatibilidad HLA en comparación con los receptores de injertos de donantes cadavéricos. Es de conocimiento que el tiempo de isquemia fría es un factor determinante en la sobrevida de los injertos ${ }^{21,22}$, de tal forma que aquellos pacientes con tiempos menores de 12 horas de isquemia fría no tendrían por qué elevar su IR y el injerto tendría mejor sobrevida; en este estudio los pacientes con menos de 12 horas de isquemia fría elevaron el IR. Acorde con publicaciones ya realizadas, los datos revelan que no es por sí sola la isquemia fría la que determina el aumento del $\mathrm{IR}^{23}$.

En cuanto al tratamiento médico inmunosupresor, se ha observado en algunos $\operatorname{artículos}^{24}$, y en este, que los esquemas de inducción no aportaron clínicamente ninguna diferencia importante sobre el IR; sin embargo, con esquemas de mantenimiento si se observó una tendencia, de los pacientes que tomaron ciclosporina, a elevar el IR, mientras que los pacientes que tomaron tacrolimus no lo hacían. Una mayor cantidad de pacientes en el brazo de IR mayor a 0.8 fueron convertidos a inhibidores de $\mathrm{M}$ - tor, y esto puede ser unas de las explicaciones de por qué la tasa de filtración glomerular, en este grupo de pacientes que empezó con una media basal de $6 \mathrm{ml} / \mathrm{min}$ menor con respecto al grupo de IR $<0.8$, se mantiene a largo plazo.

En la cohorte estudiada, los pacientes una vez se trasplantaron presentaron generalmente función inmediata del injerto independiente del IR. Acorde con estudio previo ${ }^{25}$, los pacientes que presentaron más eventos de función retardada revelaron un IR anormal, esto es explicable ya que después del impacto de la hemodiálisis las resistencias vasculares y la hemodinámia varían, alterando así el IR. Se esperaba que la función lenta del injerto predominara en los pacientes con IR anormal o de no ser así que se encontrara la misma frecuencia en los dos grupos; los datos demostraron que es mayor en los pacientes con IR normal siendo su principal causa la necrosis tubular aguda. Sin embargo, la tasa de necrosis tubular aguda fue similar para los 2 grupos, lo que lleva a inferir que quizás tenga más valor para la función lenta del injerto las complicaciones mecánicas, sin alterar el IR ya que al momento de la medición esta complicación ya está corregida. La complicación vascular se presentó en los pacientes con IR normal, fue de características venosas de tal modo que la arteria se mantuvo indemne y por tanto el IR no se afectó.

Como se ha mencionado anteriormente, existen estudios que apoyan la relación entre sobrevida de injerto con IR normal; sin embargo en los resultados de este estudio no se esperaba que al comparar la cohorte, los pacientes con IR normal aunque en poca proporción, presentaran más eventos de pérdidas de injertos que los pacientes con IR anormal. Cuando se analizan los casos de pérdida de injerto de forma específica, se observa que no existió manera de evidenciar que el IR medido en el tiempo orientara hacia su pérdida. Esto nos pone de manifiesto que no todos los pacientes con IR normal tomado al primer mes postrasplante van a desarrollar desenlaces favorables para el injerto, igualmente no todos los pacientes con IR anormal perderán por lo menos en los 3 primeros años el injerto.

En contraposición a lo dicho anteriormente en la literatura, la principal causa de muerte con injerto funcionante fue de etiología cardiovascular y el mayor porcentaje se observó en los pacientes con IR anormal $1^{2,14}$, ya que quizás el IR en este caso esté relacionado con la enfermedad aterosclerótica de base del paciente y no una lesión renal per sé.

Durante este estudio se observó que los pacientes con IR mayor de 0.8 tenían una tasa de filtración 
glomerular menor $(6 \mathrm{ml} / \mathrm{min})$ con respecto a los del grupo con IR $<0.8$, que para algunos autores puede ser de vital importancia ${ }^{26}$; sin embargo, en el seguimiento a 3 años la tasa de filtración glomerular se equilibró, esto puede explicarse a la mayor intervención terapéutica en este grupo de pacientes y lleva concluir que el IR en la población estudiada no determina el desenlace de la TFG. A 3 años de seguimiento se observó que los pacientes con IR normal tenían una proporción de pérdida de más del $50 \%$ de la TFG que los de IR anormal, esto alerta al nefrólogo porque va en contra de lo que hasta hoy se conoce, observándose que los pacientes con IR normal pueden al año deteriorar severamente su TFG; demostrando así que el IR por sí solo no es la única intervención que puede afectar la TFG del injerto, hay otras más (tiempo de isquemia fría, complicaciones quirúrgicas, esquemas de inducción y mantenimiento, infecciones, elección medicamentosa, entre otras, tabla 4). Esta afirmación indica que todas las intervenciones que se realicen para aumentar o evitar la disminución de la tasa de filtración glomerular en el transcurso del tiempo deben realizarse, ya que un paciente que tenga el IR anormal puede mejorar su TFG de forma importante, inclusive 12 meses después del trasplante. Se debe recordar que el IR se realiza al primer mes postrasplante reflejando así el estado del injerto en un solo momento; quizás si se realizaran mediciones del IR junto con los aumentos en las tasas de filtración glomerular se observaría alguna diferencia. En nuestra población es imposible asegurar que los pacientes con IR anormal estén destinados a perder el injerto o a disminuir las tasas de filtración glomerular.

La mediana de la tasa de filtración glomerular al seguimiento en el primero, segundo y tercer año fue casi similar en los 2 grupos de IR, con un promedio mayor de $60 \mathrm{ml} / \mathrm{min}$. Además, muchos de estos pacientes tenían ya cambios histológicos tempranos en la biopsia renal de protocolo, por lo que al igual que en la literatura ${ }^{26,27}$, los cambios de la tasa de filtración glomerular no se asocian en los primeros años con la presencia de hallazgos histológicos de mal pronóstico; se necesitan estudios con mayor seguimiento para ver en qué punto del seguimiento la curva de deterioro de la función renal y los hallazgos histológicos en biopsias de protocolo comienzan a correlacionarse.

Las biopsias de los injertos orientan al clínico hacia la toma de decisiones y son fuertemente útiles para

Figura 4

Aumento de la TFG más del 50\% respecto a la basal, incidencia acumulada.

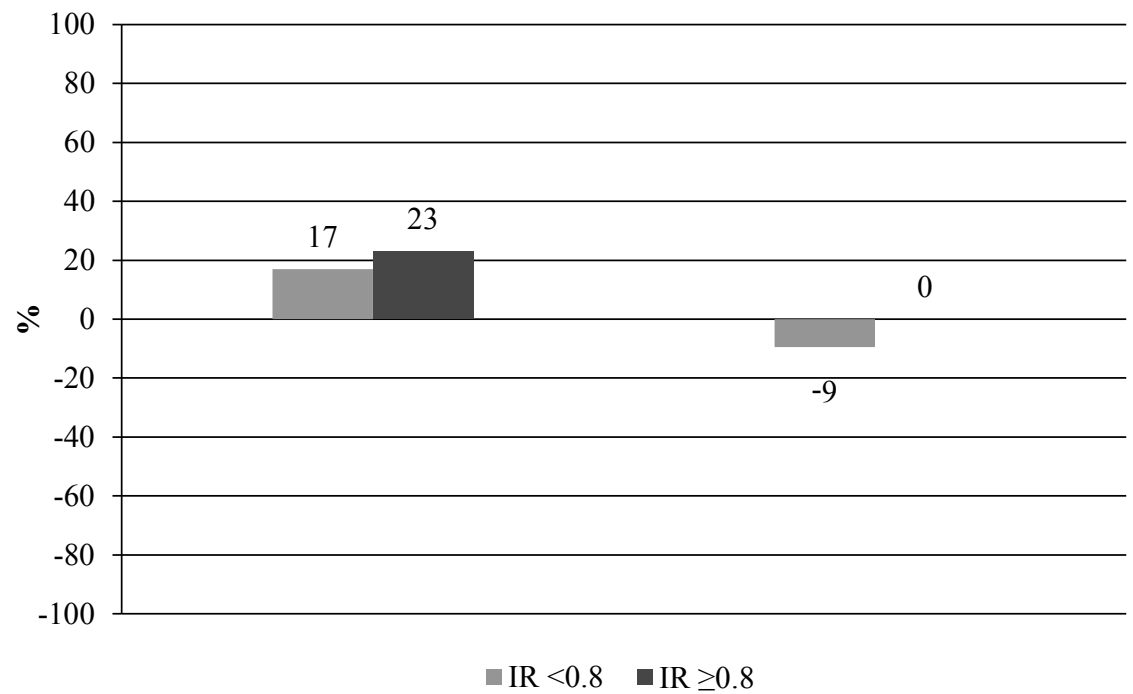


determinar el pronóstico del injerto y la nefropatía crónica. Son pocas las publicaciones que intentan correlacionar el IR con los hallazgos histológicos ${ }^{19}$. En este estudio más del $80 \%$ de la población, de los 2 grupos, fue objeto de biopsia de protocolo y a pesar de todas las medidas que se conocen para prevenir la nefropatía crónica del injerto, éste sigue siendo el hallazgo más frecuente aun con IR normal; además otros hallazgos histopatológicos de mal pronóstico como IFTA, inflamación intersticial, hialinosis arteriolar, se encontraron con igual proporción en ambos grupos. Esto nos pone de manifiesto que no todos los pacientes con IR normal van tener buenos desenlaces histológicos.

Los cambios histológicos de la biopsia del injerto renal que demuestran cronicidad como IFTA, hialinosis, se observaron en ambos grupos.

En la literatura ${ }^{28}$ se reportan diferentes puntos de corte del IR con los cambios histológicos; hemos intentado buscar en esta población de estudio el punto de corte para el IR de mayor sensibilidad y especificidad, sin embargo, dado el bajo porcentaje de desenlaces y el tiempo de seguimiento fue imposible evidenciarlo.

El rechazo inmunológico es una causa frecuente de disfunción precoz y tardía del trasplante renal; hay varios tipos de reacción inmunológica como es el rechazo celular agudo, humoral agudo y crónico del aloinjerto con demostraciones clínicas e histológicas en las biopsias renales, que requieren para su diagnóstico la presencia de anticuerpos antiHLA. Los criterios de Banff ${ }^{26}$ son criterios uniformes de aplicación a la biopsia para el diagnóstico de rechazo del injerto renal, que se actualizan periódicamente y constituyen la base para decidir el pronóstico y tratamiento. En esta población el rechazo inmunológico, en su mayoría de características subclínicas, ocurrió en igual proporción en ambos grupos de IR, al primer año. En la biopsia de protocolo al segundo año se incrementó el porcentaje en el grupo de IR $>0.8$, por lo que el rechazo se puede presentar de forma independiente al IR y su aparición puede estar más correlacionada con otros factores (Miss- match, tipo de donante, tratamiento inductor y de mantenimiento etc.).

\section{Conclusión}

Los desenlaces de los injertos no solo dependen del IR, hay otros factores que pueden influir como el tiempo de isquemia fría, esquema de tratamiento inmunosupresor de inducción y mantenimiento, el grado de incompatibilidades donante/receptor, entre otros. El aumento o la pérdida de la tasa de filtración glomerular al parecer no se correlacionan con el IR.

Cambios histopatológicos como IFTA, inflamación, hialinosis en esta población estudiada se observaron en cualquiera de los 2 grupos de IR con igual proporción, lo que sugiere que hay otros factores más fuertes que se correlacionan con la aparición de estos hallazgos histológicos.

Es necesario tener en cuenta las limitaciones del estudio; es un estudio descriptivo, con baja frecuencia de desenlaces y de seguimiento corto, por lo cual no permite evidenciar el IR como causalidad única de los desenlaces.

Solo la realización de un estudio multicéntrico, que permitan reclutar más población con análisis multivariado a futuro, podrá determinar cuál es el verdadero impacto del IR sobre la tasa de filtración glomerular del injerto a largo plazo.

Los hallazgos sugieren que la medición del IR al primer mes postrasplante es un arma importante que puede orientar al clínico con ciertos cambios anatómicos, pero que a largo plazo no predice de manera óptima desenlaces que se relacionan con la sobrevida del injerto.

\section{Agradecimientos}

Al personal de la Oficina de Trasplante Renal del Hospital San José de Bogotá, por su colaboración en la recolección de los datos.

\section{Conflicto de interés:}

Los autores declaran que no tienen conflicto de interés. 


\section{Bibiliografía}

1. Ramos E, Aoun S, Harmon WE. Expanding the donor pool: effect on graft outcome. J Am Soc Nephrol. 2002;13(10):2590-9.

2. Marsden PA. Predicting outcomes after renal transplantation--new tools and old tools. N Engl J Med. 2003;349(2):182-4.

3. Humar A, Ramcharan T, Kandaswamy R, Gillingham K, Payne WD, Matas AJ. Risk factors for slow graft function after kidney transplants: a multivariate analysis. Clin Transplant. 2002;16(6):425-9.

4. Ponticelli C. Renal transplantation 2004: where do we stand today? Nephrol Dial Transplant. 2004;19(12):2937-47.

5. Jeong JC, Ro H, Hwang YH, Lee HK, Ha J, Ahn C, et al. Cardiovascular diseases after kidney transplantation in Korea. J Korean Med Sci. 2010;25(11):1589-94.

6. Khedmat $\mathrm{H}$, Taheri S. Characteristics and prognosis of post-transplant lymphoproliferative disorders within renal allograft: Report from the PTLD.Int. Survey. Ann Transplant. 2010;15(3):80-6.

7. Arias M, Serón D, Moreso F, Bestard O, Praga M. Chronic renal allograft damage: existing challenges. Transplantation. 2011;91(9 Suppl):S4-25.

8. Radermacher J, Mengel M, Ellis S, Stuht S, Hiss M, Schwarz A, et al. The renal arterial resistance index and renal allograft survival. N Engl J Med. 2003;349(2):115-24.

9. de Vries AP, van Son WJ, van der Heide JJ, Ploeg RJ, Navis G, de Jong PE, et al. The predictive value of renal vascular resistance for late renal allograft loss. Am J Transplant. 2006;6(2):364-70.

10. Krumme B, Grotz W, Kirste G, Schollmeyer P, Rump LC. Determinants of intrarenal Doppler indices in stable renal allografts. J Am Soc Nephrol. 1997;8(5):813-6.

11. Goldfarb DA. The natural history of chronic allograft nephropathy. J Urol. 2005;173(6):2106.

12. Heine GH, Gerhart MK, Ulrich C, Köhler H, Girndt M. Renal Doppler resistance indices are associated with systemic atherosclerosis in kidney transplant recipients. Kidney Int. 2005;68(2):878-85.

13. Nezami N, Tarzamni MK, Argani H, Nourifar M. Doppler ultrasonographic indexes in kidney transplant recipients: its relationship with kidney function. Iran J Kidney Dis. 2007;1(2):82-7.

14. Łebkowska U, Malyszko J, Łebkowski W, Brzósko S, Kowalewski R, Łebkowski T, et al. The predictive value of arterial renal blood flow parameters in renal graft survival. Transplant Proc. 2007;39(9):2727-9.

15. Saracino A, Santarsia G, Latorraca A, Gaudiano V. Early assessment of renal resistance index after kidney transplant can help predict long-term renal function. Nephrol Dial Transplant. 2006;21(10):2916-20.

16. Akgul A, Ibis A, Sezer S, Basaran C, Usluogullari A, Ozdemir FN, et al. Early assessment of renal resistance index and long-term renal function in renal transplant recipients. Ren Fail. 2009;31(1):18-24.

17. Bergmann IP, Böger RH, Marti E, Frey FJ, Schwedhelm E, Eisenberger U. Renal resistance index in renal allograft recipients: a role for ADMA. Am J Kidney Dis. 2009;54(2):327-33.

18. Vallejos A, Alperovich G, Moreso F, Cañas C, de Lama ME, Gomà M, et al. Resistive index and chronic allograft nephropathy evaluated in protocol biopsies as predictors of graft outcome. Nephrol Dial Transplant. 2005;20(11):2511-6.

19. Kirkpantur A, Yilmaz R, Abali G, Arici M, Altun B, Aki T, et al. Utility of c2 monitoring in prediction of diastolic dysfunction in renal transplant recipients. Transplant Proc. 2008;40(1):171-3.

20. Khosroshahi HT, Heris HK, Makhdami N, Habibzadeh A, Badrogli N, Oskoii R, et al. Time-dependent Doppler ultrasonographic findings in transplanted kidneys from living donors: a 5-year follow-up study. Transplant Proc. 2011;43(2):482-4.

21. Salahudeen AK, May W. Reduction in cold ischemia time of renal allografts in the United States over the last decade. Transplant Proc. 2008;40(5):1285-9. 
22. Gencoglu EA, Ayaz S, Moray G, Emiroglu R, Haberal M. Effect of prolonged cold ischemia time on the outcome of cadaveric renal grafts. Transplant Proc. 2003;35(7):2564-5.

23. Tranquart F, Lebranchu Y, Haillot O, Pourcelot D, Grezard O, Pourcelot L. The use of perioperative Doppler ultrasound as a screening test for acute tubular necrosis. Transpl Int. 1993;6(1):14-7.

24. Liu Y, Zhou P, Han M, Xue CB, Hu XP, Li C. Basiliximab or antithymocyte globulin for induction therapy in kidney transplantation: a meta-analysis. Transplant Proc. 2010;42(5):1667-70.

25. Chudek J, Kolonko A, Król R, Ziaja J, Cierpka L, Wiecek A. The intrarenal vascular resistance parameters measured by duplex Doppler ultrasound shortly after kidney transplantation in patients with immediate, slow, and delayed graft function. Transplant Proc. 2006;38(1):42-5.

26. Budde K, Lehner F, Sommerer C, Arns W, Reinke P, Eisenberger U, et al. Conversion from cyclosporine to everolimus at 4.5 months posttransplant: 3-year results from the randomized ZEUS study. Am J Transplant. 2012;12(6):1528-40.

27. Elgueta S, Fuentes C, Arenas A, Labraña C, Gajardo JG, López M, et al. Evaluation of the stages involved in cold ischemia time in renal transplants in Chile. Transplant Proc. 2010;42(1):248-9.

28. Cazap E, Buzaid A, Garbino C, de la Garza J, Orlandi F, Schwartsmann G, et al. Breast cancer in Latin America: experts perceptions compared with medical care standards. Breast. 2010;19(1):50-4. 